\title{
Triage of high-risk HPV-positive women in population-based screening by miRNA expression analysis in cervical scrapes; a feasibility study
}

Iris Babion ${ }^{1}$, Barbara C. Snoek ${ }^{1}$, Putri W. Novianti ${ }^{1,2}$, Annelieke Jaspers $^{1}$, Nienke van Trommel ${ }^{3}$, Daniëlle A. M. Heideman' ${ }^{1}$, Chris J. L. M. Meijer ${ }^{1}$, Peter J. F. Snijders' ${ }^{1}$, Renske D. M. Steenbergen ${ }^{1 *}$ and Saskia M. Wilting ${ }^{4}$

\begin{abstract}
Background: Primary testing for high-risk HPV (hrHPV) is increasingly implemented in cervical cancer screening programs. Many hrHPV-positive women, however, harbor clinically irrelevant infections, demanding additional disease markers to prevent over-referral and over-treatment. Most promising biomarkers reflect molecular events relevant to the disease process that can be measured objectively in small amounts of clinical material, such as miRNAs. We previously identified eight miRNAs with altered expression in cervical precancer and cancer due to either methylation-mediated silencing or chromosomal alterations. In this study, we evaluated the clinical value of these eight miRNAs on cervical scrapes to triage hrHPV-positive women in cervical screening.

Results: Expression levels of the eight candidate miRNAs in cervical tissue samples $(n=58)$ and hrHPV-positive cervical scrapes from a screening population $(n=187)$ and cancer patients $(n=38)$ were verified by quantitative RT-PCR. In tissue samples, all miRNAs were significantly differentially expressed $(p<0.05)$ between normal, highgrade precancerous lesions (CIN3), and/or cancer. Expression patterns detected in cervical tissue samples were reflected in cervical scrapes, with five miRNAs showing significantly differential expression between controls and women with CIN3 and cancer. Using logistic regression analysis, a miRNA classifier was built for optimal detection of CIN3 in hrHPV-positive cervical scrapes from the screening population and its performance was evaluated using leave-one-out cross-validation. This miRNA classifier consisted of miR-15b-5p and miR-375 and detected a major subset of CIN3 as well as all carcinomas at a specificity of 70\%. The CIN3 detection rate was further improved by combining the two miRNAs with HPV16/18 genotyping. Interestingly, both miRNAs affected the viability of cervical cancer cells in vitro.
\end{abstract}

Conclusions: This study shows that miRNA expression analysis in cervical scrapes is feasible and enables the early detection of cervical cancer, thus underlining the potential of miRNA expression analysis for triage of hrHPV-positive women in cervical cancer screening.

Keywords: miRNA, HPV, Cervical cancer, CIN, Scrape, Triage, Screening, qRT-PCR

\footnotetext{
* Correspondence: r.steenbergen@vumc.nl

'Cancer Center Amsterdam, Department of Pathology, VU University Medical

Center, Amsterdam, The Netherlands

Full list of author information is available at the end of the article
}

(c) The Author(s). 2018 Open Access This article is distributed under the terms of the Creative Commons Attribution 4.0 International License (http://creativecommons.org/licenses/by/4.0/), which permits unrestricted use, distribution, and reproduction in any medium, provided you give appropriate credit to the original author(s) and the source, provide a link to the Creative Commons license, and indicate if changes were made. The Creative Commons Public Domain Dedication waiver (http://creativecommons.org/publicdomain/zero/1.0/) applies to the data made available in this article, unless otherwise stated. 


\section{Background}

Cervical cancer screening by cytological examination of cervical scrapes has largely reduced the incidence and mortality rates of cervical cancer in developed countries due to early detection of well-recognizable and treatable precursor lesions (cervical intraepithelial neoplasia (CIN), graded 1-3) [1]. Persistent infection with high-risk types of the human papillomavirus (hrHPV) is a necessary cause of cervical cancer [2-4]. Testing for hrHPV DNA has been demonstrated to have a higher sensitivity for the detection of cervical high-grade CIN and cancer than the cytology-based Pap smear $[5,6]$. Consequently, hrHPV testing has recently been implemented as primary screening method in The Netherlands and various other countries. As hrHPV testing also detects women with clinically irrelevant transient infections, additional triage markers are required to identify women with high-grade CIN lesions who are in need of treatment given their risk of developing cancer. Cytology is the currently recommended triage strategy for hrHPV-positive women. An ideal triage test, however, should be objective (non-morphological), available in a high-throughput format and feasible in low-resource countries. For this purpose, HPV16/18 genotyping has previously been investigated as triage test in cervical cancer screening [7-9]. Additional molecular markers reflecting the underlying carcinogenic process are highly appealing alternatives. These include DNA copy number aberrations and DNA methylation changes in the host cell genome that result in altered coding and non-coding gene expression [3].

MicroRNAs (miRNAs) belong to an abundant class of small non-coding RNAs that post-transcriptionally regulate gene expression [10]. Altered expression of miRNAs has been shown to contribute to human malignancies, including cervical cancer, by influencing expression of oncogenes and tumor suppressor genes and subsequent deregulation of important intracellular pathways (reviewed by [11]). Given their short length of approximately 22 nucleotides, miRNAs are stable biomolecules and are less prone to degradation than their longer counterparts such as mRNAs or long non-coding RNAs [10, 12, 13]. It is therefore not surprising that miRNAs have been suggested and investigated as biomarkers for cancer diagnostics and prognostics [14-16].

To identify miRNAs that are relevant to cervical carcinogenesis, we previously performed an integrative screen combining array-based miRNA expression profiles with DNA copy number aberrations and DNA methylation $[17,18]$. This resulted in the identification of five miRNAs for which differential expression was associated with frequently observed chromosomal gains of chromosomes 1q and 3q (miR-9-5p, miR-15b-5p,
miR-28-5p) or losses of chromosome 11q (miR-100-5p, miR-125b-5p), and three miRNAs (miR-149-5p, miR203a-3p, miR-375) for which gene silencing was mediated by DNA methylation of their respective promoter sequences (Table 1). Functional studies for miR-9, miR-203a, and miR-375 supported the biological relevance of these miRNA alterations, which were found to be involved in proliferation and anchorage independence of HPV-transformed cells [17-19].

In this study, we evaluated the clinical value of the eight either genetically or epigenetically deregulated miRNAs to serve as triage markers on cervical scrapes of hrHPV-positive women in cervical screening. For this purpose, we verified expression of the discovered $8 \mathrm{miR}$ NAs in 58 cervical tissues and archival cervical scrapes of $225 \mathrm{hrHPV}$-positive women, built a predictive miRNA classifier, and evaluated its performance for the detection of high-grade CIN and cancer using leave-one-out cross-validation and ROC curve analysis.

\section{Methods \\ Clinical specimens}

Cervical tissue samples consisted of microdissected fresh frozen specimens, of which the majority has previously been used for miRNA microarray analysis [17]. In total, 8 normal cervical epithelial samples, 18 high-grade cervical intraepithelial neoplasia (CIN2-3) lesions, 22 cervical squamous cell carcinomas (SCC), and 11 adenocarcinomas (AC) were included. All but one normal sample were hrHPV-positive. The median age per group was as follows: normal, 35 years (range 31-47); CIN2-3, 34 years (range 26-54); SCC, 48.5 years (range 25-78); and AC, 39 years (range 31-64).

Cervical scrapes of $66 \mathrm{hrHPV}$-positive women without underlying disease (Pap 1) and 121 women with CIN3 were obtained from a screening population in the Utrecht region that had been collected between January 2010 and December 2011. Original $20 \mathrm{ml}$ samples were

Table 1 Candidate miRNAs

\begin{tabular}{|c|c|c|c|}
\hline miRNA & Regulation [17] & $\begin{array}{l}\text { Potential regulation } \\
\text { mechanism }[17,18]\end{array}$ & Class [17] \\
\hline miR-9-5p & Up & Chromosomal gain (1q) & Late \\
\hline miR-15b-5p & Up & Chromosomal gain (3q) & Late \\
\hline $\operatorname{miR}-28-5 p$ & Up & Chromosomal gain (3q) & $\begin{array}{l}\text { Early } \\
\text { continuous }\end{array}$ \\
\hline miR-100-5p & Down & Chromosomal loss (11q) & Late \\
\hline miR-125b-5p & Down & Chromosomal loss (11q) & Late \\
\hline miR-149-5p & Down & DNA methylation & $\begin{array}{l}\text { Early } \\
\text { continuous }\end{array}$ \\
\hline miR-203a-3p & Down & DNA methylation & $\begin{array}{l}\text { Early } \\
\text { continuous }\end{array}$ \\
\hline miR-375 & Down & DNA methylation & Late \\
\hline
\end{tabular}


concentrated and stored in $1 \mathrm{ml}$ ThinPrep medium (Hologic, Vilvoorde, Belgium) at $-80{ }^{\circ} \mathrm{C}$. For most samples, HPV genotyping was performed using the general primer GP5+/6+-mediated PCR-enzyme immunoassay in combination with the luminex genotyping kit HPV GP at the time of sample collection [20,21]. For samples with sufficient amounts of DNA for which no previous genotyping results were available, we used the HPV-Risk Assay (Self-screen BV, Amsterdam, The Netherlands) to complete our dataset [22]. Women without disease had a median age of 41 years (range 21-61). The median age of women with CIN3 was 35 years (range 22-60). HrHPV-positive scrapes from women with underlying cervical SCC $(n=29)$ and AC $(n=9$, consisting of 7 AC and 2 adenosquamous carcinomas) [23, 24] were collected at the Antoni van Leeuwenhoek Hospital Amsterdam, The Netherlands, between January 2015 and March 2017. All cervical cancer samples were tested for hrHPV using the HPV-Risk Assay. Women with SCC had a median age of 51 years (range 29-86), and the median age of women with AC was 45 years (range 27-62).

\section{RNA isolation}

Total RNA was isolated using TRIzol reagent (Thermo Fisher Scientific, Landsmeer, The Netherlands) according to the manufacturer's instructions. The $\mathrm{Qubit}^{\circ}$ microRNA Assay kit was used to quantify small RNA concentrations on a Qubit $^{\circ} 2.0$ Fluorometer (both ThermoFisher Scientific).

\section{Quantitative RT-PCR}

Expression of hsa-miR-9-5p, hsa-miR-15b-5p, hsamiR-28-5p, hsa-miR-100-5p, hsa-miR-125b-5p, hsa-miR -149-5p, hsa-miR-203a-3p, and hsa-miR-375 was measured using TaqMan microRNA assays (000583, 000390, 000411, 000437, 000449, 002255, 000507, 000564; Thermo Fisher Scientific). For cervical scrapes, RNU24, RNU43, U6, U75, hsa-miR-423-3p, and hsamiR-425-5p were included as potential reference genes (001001, 001095, 001973, 001219, 002626, 001516; Thermo Fisher Scientific).

Reverse transcription (RT) of all targets was multiplexed and validated in comparison to singleplex RT reactions (data not shown). In short, a primer pool was created by combining the specific RT primers. cDNA was synthesized from $20 \mathrm{ng}$ small RNA template if available, for 5 samples the maximum possible amount $(<$ $20 \mathrm{ng}$ ) of RNA was used. Each $16 \mu$ reaction contained $6 \mu \mathrm{l}$ primer pool, $0.3 \mu \mathrm{l}$ dNTPs $(100 \mathrm{mM}), 1.5 \mu \mathrm{l}$ RT buffer, $0.19 \mu \mathrm{l}$ RNase inhibitor $(20 \mathrm{U} / \mu \mathrm{l})$, and $3 \mu \mathrm{l}$ MultiScribe Reverse Trancriptase (TaqMan microRNA Reverse Transcription kit, Thermo Fisher Scientific). Quantitative PCR reactions were performed on the $\mathrm{ViiA}^{\mathrm{m}}$ 7 Real-Time PCR System (Thermo Fisher Scientific) in a 384-well format. Each $10 \mu \mathrm{l}$ reaction consisted of $5 \mu \mathrm{l}$
$\mathrm{TaqMan}^{\bullet}$ Universal Master Mix II, $0.5 \mu \mathrm{l}$ miRNA specific TaqMan assays (Thermo Fisher Scientific), $3.5 \mu \mathrm{l}$ $\mathrm{H}_{2} \mathrm{O}$, and $1 \mu \mathrm{l}$ cDNA. Cycle conditions for cDNA synthesis and PCR were used according to the manufacturer's protocols.

RNU24 and miR-423-3p were selected for normalization in cervical tissue samples and scrapes using our previously published strategy (data not shown) [25]. Data were normalized to the geometric mean $\mathrm{Ct}$ of both reference genes applying the $2^{-\Delta C_{t}}$ method [26]. All samples had a reference gene geometric mean $\mathrm{Ct} \leq 32$ and were therefore considered to be suitable for miRNA expression analysis.

\section{Statistical analysis}

Statistical analysis was performed using $\mathrm{R}$ version 3.1.2. For logistic regression, $\mathrm{R}$ packages $\mathrm{pROC}$ and GRridge were used. The Spearman correlation coefficient (Rho) and associated $p$ value was calculated to assess the agreement between qRT-PCR and previous microarray results. We performed an omnibus Kruskal-Wallis test to compare miRNA expression levels between normal, CIN3, SCC, and AC for each marker. Further, Wilcoxon rank test was applied with a significance level of 0.05 (two-sided) when the omnibus test showed a significant result $(p<0.05) . p$ values from the post-hoc test were corrected with Benjamini-Hochberg correction method. Individual miRNA models and multi-miRNA classifiers for the detection of CIN3 were built performing univariable and multivariable logistic regression on square root transformed $\mathrm{Ct}$ ratios and evaluated using leave-one-out cross-validation. As a result, predicted probabilities, i.e., values between 0 and 1 representing the risk of an underlying CIN3, were calculated for each sample. For the construction of multi-miRNA classifiers, multivariable logistic regression analysis was followed by backward elimination to select relevant markers. Receiver-operated characteristic (ROC) curve analysis was carried out to evaluate the performance of the miRNA classifiers in detecting CIN3. For comparison of the obtained AUCs with a random classifier with AUC $=0.5$, DeLong's test for two correlated ROC curves was used [27].

\section{Cell culture, transfection, and cell viability assay of cervical cancer cell lines}

Cervical cancer cell lines $\mathrm{SiHa}$ and CaSki were authenticated by STR testing using the Powerplex16 System (Promega, Leiden, The Netherlands) and cultured as described previously [28]. Cells were transiently transfected with $30 \mathrm{nM}$ miRCURY LNA microRNA Power inhibitors for miR-15b-5p and negative control A (4103019, 199006; Exiqon, Vedbaek, Denmark) or $30 \mathrm{nM}$ miRIDIAN microRNA mimics for miR-375 and negative control \#2 (C-300682-05, CN-002000-01; GE 
Dharmacon, Lafayette, CO, USA). Cells were transfected with Dharmafect 1 (GE Dharmacon) for at least $6 \mathrm{~h}$ according to the manufacturer's instructions. After transfection, cells were seeded in triplicate in 96-well plates (2500 cells/well). Cell viability was measured using the fluorometric CellTiter-Blue assay (Promega, Madison, WI, USA) according to the manufacturer's protocol at days 0 and 2. The average measurement of day 0 was subtracted from the measurements at day 2. Each experiment was performed at least two times.

\section{Results}

Microarray-based differential miRNA expression in cervical tissue specimens can be verified by qRT-PCR

To confirm our previously obtained microarray results, we used qRT-PCR to determine the expression levels of the eight genetically or epigenetically deregulated miRNAs in normal cervical squamous epithelium $(n=8)$, high-grade CIN lesions (CIN2-3, $n=18), \operatorname{SCC}(n=22)$, and $\mathrm{AC}(n=11)$ tissue specimens, of which 44 had also been analyzed by microarray [17]. Except for miR-28-5p and miR-100-5p (Spearman correlation coefficient (Rho) $=0.521$ and $\mathrm{Rho}=0.645$, respectively), qRT-PCR results strongly correlated with microarray results as indicated by Rho $>0.75$ (Additional file 1: Figure S1). Because of the low correlations observed for miR-28-5p and miR-100-5p, both miRNAs were excluded from further analysis. Significantly differential expression between normal and CIN2-3 could be verified for 1 out of 2 upregulated miRNAs (miR-9-5p) and for 2 out of 4 downregulated miRNAs (miR-149-5p, miR-203a-3p; Additional file 2: Figure S2 and Additional file 3: Table S1). Downregulation of miR-375 in CIN2-3 compared to normal was borderline significant $(p=0.067)$. All miRNAs showed significantly differential expression between normal and SCC.

\section{Differential miRNA expression is reflected in cervical scrapes}

Next we analyzed whether altered expression of candidate miRNAs is also detectable in cervical scrapes. HrHPV-positive scrapes of women without cervical disease (normal, $n=66)$ or with underlying CIN3 $(n=121)$, SCC $(n=29)$, or AC $(n=9)$ were analyzed by qRT-PCR. All six miRNAs except for miR-9-5p could be detected in at least $99 \%$ of samples. MiR-9-5p remained undetected in about one fourth $(23 \%, 49 / 212)$ of samples. Low expression levels of miR-9-5p were consistent with microarray and qRT-PCR results obtained in cervical tissue specimens (Fig. 1, Additional file 2: Figure S2). Because reproducibility is reduced and technical PCR noise increases when amplifying lowly abundant transcripts [29-31], we did not consider miR-9-5p a suitable biomarker and excluded it from further analysis. MiRNA expression results obtained in hrHPV-positive cervical scrapes were generally comparable to those obtained in tissue samples (Fig. 1, Additional file 2: Figure S2 and Additional file 4: Table S2). Expression of miR-15b-5p was significantly increased in scrapes of women with CIN3 compared to controls, while miR-125b-5p and miR-375 were significantly downregulated in scrapes of women with CIN3. No significant difference between normal and CIN3 was observed for miR-149-5p and miR-203a-3p. Similar to observations in tissue samples, the largest expression change between scrapes of women with CIN3 and SCC was observed for upregulated miR-15b-5p. Expression of miR-149-5p and miR-375 was significantly decreased in scrapes of women with SCC compared to normal and CIN3. Comparison of normal controls to AC showed a significant increase in expression of all upregulated miRNAs in scrapes of AC patients. In accordance with tissue results, miR-125b-5p and miR-149-5p were significantly downregulated between normal and $\mathrm{AC}$, and miR-375 did not show a significant difference between normal scrapes and those of women with AC.

\section{Predictive miRNA classifier detects large subset of CIN3 lesions}

Univariate logistic regression analysis was carried out on expression results of the five remaining markers (miR-15b-5p, miR-125b-5p, miR-149-5p, miR-203a-3p and miR-375) obtained from hrHPV-positive cervical scrapes of women without cervical disease and women with underlying CIN3, and validated by leave-one-out cross-validation. Single miRNAs achieved areas under the curve (AUC) varying from 0.523 (miR-203a-3p) to 0.605 (miR-125b-5p, Table 2, Fig. 2a).

To determine the most discriminative miRNA marker panel for CIN3, we performed multivariable logistic regression analysis followed by backward marker selection and validated results by leave-one-out cross-validation. This resulted in a 2-miRNA classifier consisting of miR-15b-5p and miR-375 with an AUC of 0.622 and optimal sensitivity and specificity of 55 and $70 \%$, respectively (Table 2, Fig. 2b). Adding additional miRNAs to the 2-marker panel did not further improve performance.

\section{MiRNA classifier detects all cervical carcinomas}

To test how our miRNA classifiers performed in the detection of cervical carcinomas, we applied the previously determined regression models and corresponding cutoffs to our results obtained on cervical scrapes of women with underlying SCC or AC. The 2-miRNA classifier detected all SCC and all AC (Table 3). Overall, the 2-miRNA classifier achieved a CIN3+ detection rate of $65 \%$ at $70 \%$ specificity. 


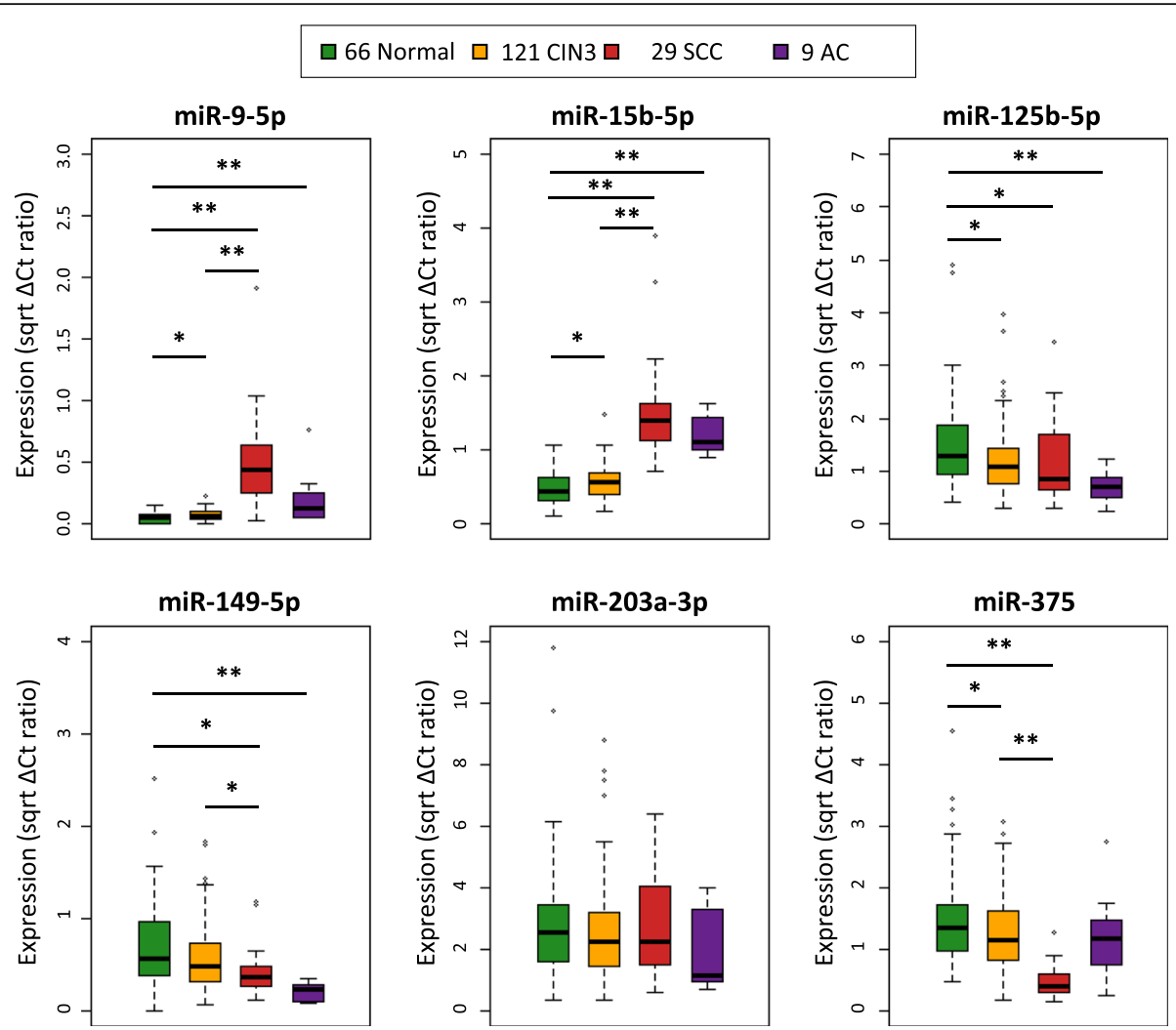

Fig. 1 Differential expression of selected miRNAs in cervical scrapes. qRT-PCR results were normalized to RNU24 and miR-423, and all values were square root transformed. ${ }^{*} p<0.05,{ }^{* *} p<0.005$

HPV16/18 genotyping in conjunction with our miRNA classifier improves CIN3 detection

To compare the performance of our 2-miRNA classifier to HPV16/18 genotyping, samples were either classified as HPV16/18 positive (19 normal, 71 CIN3) or other hrHPV type positive (46 normal, 37 CIN3), for those samples for which the genotype was known ( $n=173$ out of 187). For this smaller sample set, we built (i) a new 2-miRNA classifier consisting of miR-15b and miR-375 and (ii) a logistic regression model combining the 2-miRNA classifier with HPV16/18 genotyping. Consistent with previous reports, HPV16/18 genotyping achieved $66 \%$ sensitivity and $68 \%$ specificity for CIN3 detection (Table 4) [7, 32]. The 2-miRNA classifier obtained in the smaller sample set had a comparable performance to the one obtained from the entire set of samples (Tables 3 and 4). While HPV16/18 genotyping alone was inferior to the 2-miRNA classifier $(p=5.2$

Table 2 Comparison of optimal sensitivity and specificity between miRNA panels for the detection of CIN3 based on leave-one-out cross-validation

\begin{tabular}{|c|c|c|c|c|c|}
\hline Panel & AUC & Cutoff & Sensitivity (\%) & Specificity (\%) & $p$ value ${ }^{*}$ \\
\hline \multicolumn{6}{|l|}{ Single markers } \\
\hline miR-15b-5p & 0.573 & 0.629 & 62.0 & 56.1 & 0.098 \\
\hline miR-125b-5p & 0.605 & 0.641 & 72.7 & 47.0 & 0.020 \\
\hline miR-149-5p & 0.542 & 0.597 & 84.3 & 28.8 & 0.356 \\
\hline miR-203a-3p & 0.523 & 0.654 & 62.0 & 48.5 & 0.619 \\
\hline miR-375 & 0.565 & 0.671 & 52.9 & 62.1 & 0.145 \\
\hline \multicolumn{6}{|l|}{ Two markers } \\
\hline miR-15b-5p/375 & 0.622 & 0.682 & 54.5 & 69.7 & 0.006 \\
\hline
\end{tabular}

${ }^{*} p$ value: comparison between the miRNA classifier and a random classifier with an AUC of 0.5 

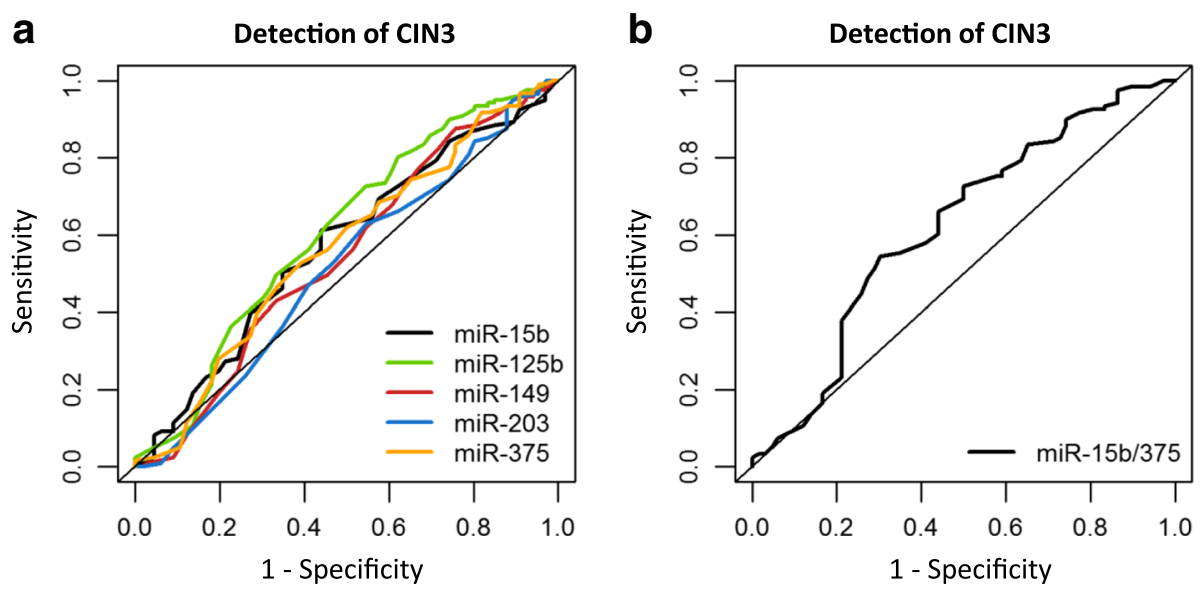

Fig. 2 ROC curve analysis of miRNA classifiers for the detection of CIN3. Results obtained from 66 hrHPV-positive scrapes from women without underlying disease and 121 scrapes from women with CIN3 were used to build (a) individual miRNA classifiers and (b) a 2-miRNA classifier. Classifiers were validated by leave-one-out cross-validation

e-16, Fig. 3), a classifier combining our two selected miRNAs with HPV16/18 genotyping had an improved performance and achieved an AUC of 0.712 (Table 4, Fig. 3). The 2-miRNA classifier adjusted by HPV16/18 type had a significantly better performance than the 2-miRNA classifier $(p=0.011)$. Including HPV16/18 genotyping in the classifier increased both sensitivity and specificity to 63 and 77\%, respectively, and all SCC and $\mathrm{AC}$ were detected (data not shown).

Knockdown of miR-15b-5p and ectopic expression of miR375 reduces viability in cervical cancer cells

Next, we investigated whether our miRNA markers are directly involved in cervical carcinogenesis, as is suggested by their genetic (miR-15b-5p) or epigenetic (miR-375) regulation. Transfection of cervical cancer cell lines $\mathrm{SiHa}$ and CaSki with miR-15b-5p inhibitors led to a reduction in cell viability, suggesting that miR-15b-5p acts as an oncomiR (Fig. 4a). Ectopic expression of

Table 3 Sensitivity of miRNA panels for the detection of SCC and $A C$

\begin{tabular}{lll}
\hline Panel & \multicolumn{2}{l}{ Sensitivity \% } \\
\cline { 2 - 3 } & Detection of SCC & Detection of AC \\
\hline Single markers & & 100 \\
miR-15b-5p & 100 & 100 \\
miR-125b-5p & 69.0 & 100 \\
miR-149-5p & 93.1 & 66.7 \\
miR-203a-3p & 55.2 & 55.6 \\
miR-375 & 96.6 & \\
Two markers & & 100 \\
miR-15b-5p/375 & 100 & \\
\hline
\end{tabular}

SCC squamous cell carcinoma, $A C$ adenocarcinoma
miR-375 in SiHa and CaSki cells significantly reduced cell viability, supporting its role as tumor suppressive miRNA (Fig. 4b).

\section{Discussion}

In this study, we analyzed the triage capacity on hrHPV-positive cervical scrapes of a panel of miRNAs that exhibit either genetically or epigenetically mediated expression changes in cervical precancerous and cancerous tissue specimens and that in part have also been shown to be functionally involved in cervical carcinogenesis $[17,18]$. We found that expression patterns detected in cervical tissue samples were reflected in cervical scrapes. By logistic regression analysis, a 2-miRNA classifier was built that at $70 \%$ specificity achieved $55 \%$ sensitivity for the detection of CIN3 and 100\% sensitivity for the detection of SCC and AC. Upon inclusion of HPV16/18 genotyping, the sensitivity and specificity for CIN3 detection could be increased to 63 and 77\%, respectively. Our data suggest that miRNA expression analysis offers a promising alternative molecular tool to triage hrHPV-positive women.

In a systematic review, Sharma et al. identified a total of 246 miRNAs that become deregulated in cervical cancer, with miR-21, miR-143, miR-145, miR-203, miR-214, and miR-218 being the most frequently described [33]. Most published studies identified deregulated miRNAs in cervical tissue samples by microarray analysis $[17,34,35]$. Studies focusing on the diagnostic or prognostic use of miRNAs detected by qRT-PCR in cervical biopsies are numerous, as are studies investigating the functional contribution of individual miRNAs to cervical cancer development. Data on the clinical applicability of miRNA expression analysis in cervical scrapes for cervical screening purposes, however, is limited. To 
Table 4 Optimal sensitivity and specificity for the detection of CIN3 for hrHPV type (HPV16/18, others) and the miRNA classifier in conjunction with hrHPV type based on a smaller sample set with known hrHPV type infection and leave-one-out cross-validation

\begin{tabular}{|c|c|c|c|c|c|}
\hline Panel & AUC & Cutoff & Sensitivity \% & Specificity \% & $p$ value $^{*}$ \\
\hline \multicolumn{6}{|l|}{ Single marker } \\
\hline HPV type & 0.445 & n.a. & 65.7 & 67.7 & 0.266 \\
\hline \multicolumn{6}{|l|}{ Multiple markers } \\
\hline miR-15b-5p/375 & 0.622 & 0.656 & 55.6 & 69.2 & 0.008 \\
\hline miR-15b-5p/375/HPV & 0.712 & 0.666 & 63.0 & 76.9 & 5.8 e-07 \\
\hline
\end{tabular}

" $p$ value: comparison between the miRNA classifier and a random classifier with an AUC of 0.5

CIN cervical intraepithelial neoplasia, AUC area under the curve, n.a not applicable

the best of our knowledge, only Tian et al. published on the analysis of a panel of candidate miRNA markers in HPV-positive scrapes of a gynecology outpatient clinic population to date [36]. Candidate miRNAs analyzed by Tian et al. have previously been shown to become differentially expressed during disease progression. A combination of miR-375 and miR-424 resulted in an AUC value of 0.853 for the detection of CIN3+, showing the promise of biologically relevant miRNAs as disease markers. Importantly, our study as well as the study by Tian et al. shows that the use of more than one miRNA improves detection of cervical disease. Although miR-15b-5p alone detected all carcinomas, combining miR-15b-5p with miR-375 proved beneficial for the

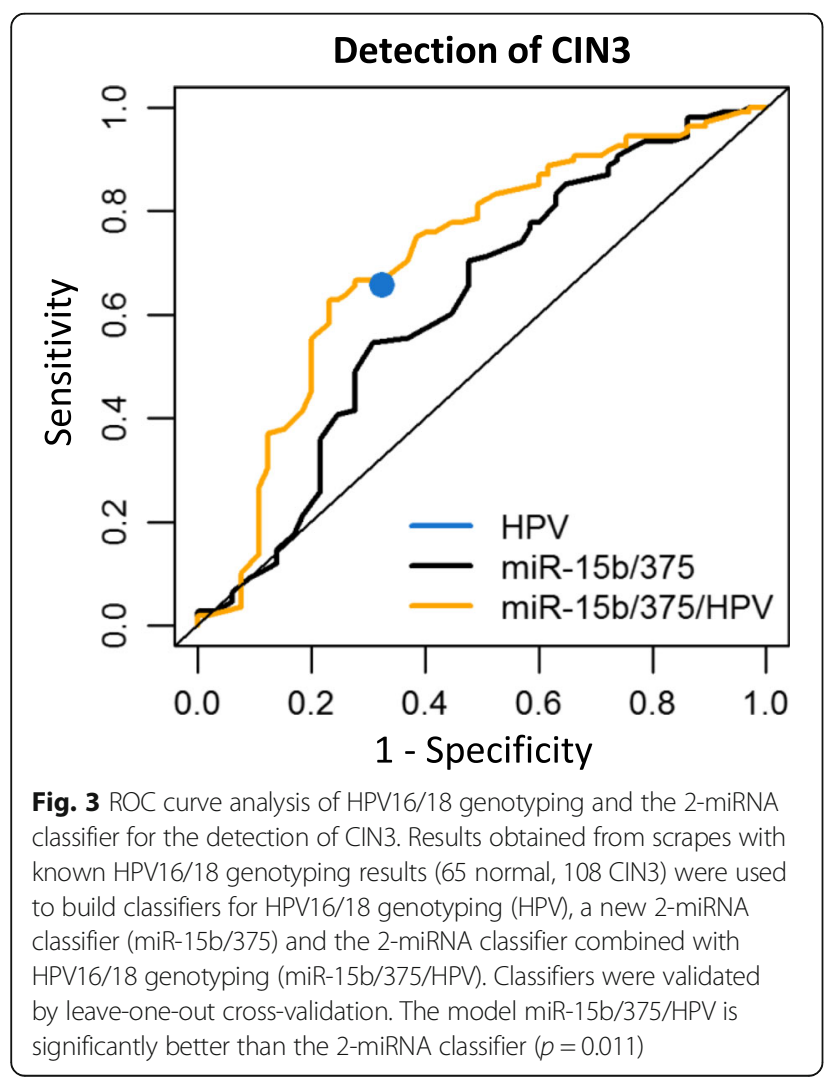

detection of CIN3 without a loss of sensitivity for SCC and $\mathrm{AC}$. The fact that the individual miRNA performing best in the detection of CIN3 (miR-125b-5p) was not included in the 2-miRNA classifier further demonstrates that analysis of multiple complementary miRNAs can improve the detection of cervical disease. Importantly, we here show that combining miRNA profiling with HPV16/18 genotyping can further improve the detection of cervical precancer. This is especially attractive, as HPV16/18 genotyping is frequently included in clinically validated HPV tests [20,37] and HPV16/18 genotyping and miRNA expression analysis can be performed on the same cervical scrape.

In line with Tian et al., our study shows that downregulated miRNAs can be suitable biomarkers, although the selection of downregulated markers may seem counterintuitive at first. It is important to note that the relative decrease in expression observed with cervical disease progression does not give an indication of the absolute abundance of a miRNA. Differences in performance between the study of Tian et al. and ours are most likely due to differences between study populations. While our cohort of cervical scrapes was obtained from a screening population, Tian et al. analyzed scrapes from a clinic-based referral population, which potentially contains more advanced CIN lesions. Altered expression of our candidate miRNAs is caused by either genetic or epigenetic changes which have previously been shown to be associated with cervical cancer and so-called advanced CIN3 lesions [3]. Their association with progression risk to cancer could explain why our miRNA classifiers detect only a subset of CIN3 lesions. Our 2-miRNA classifier detected all cervical cancers and 55\% of CIN3 at $70 \%$ specificity, a generally accepted specificity for triage markers [38]. At present, adopted triage options for HPV-positive women include reflex cytology, HPV16/18 genotyping, repeat HPV testing, and/or repeat cytology. While triage by the current miRNA panel does not yet meet the criteria for acceptability of a triage strategy [39], we here show that triage by miRNA expression analysis is feasible and offers a promising alternative. MiRNA analysis is objective, highly reproducible, 

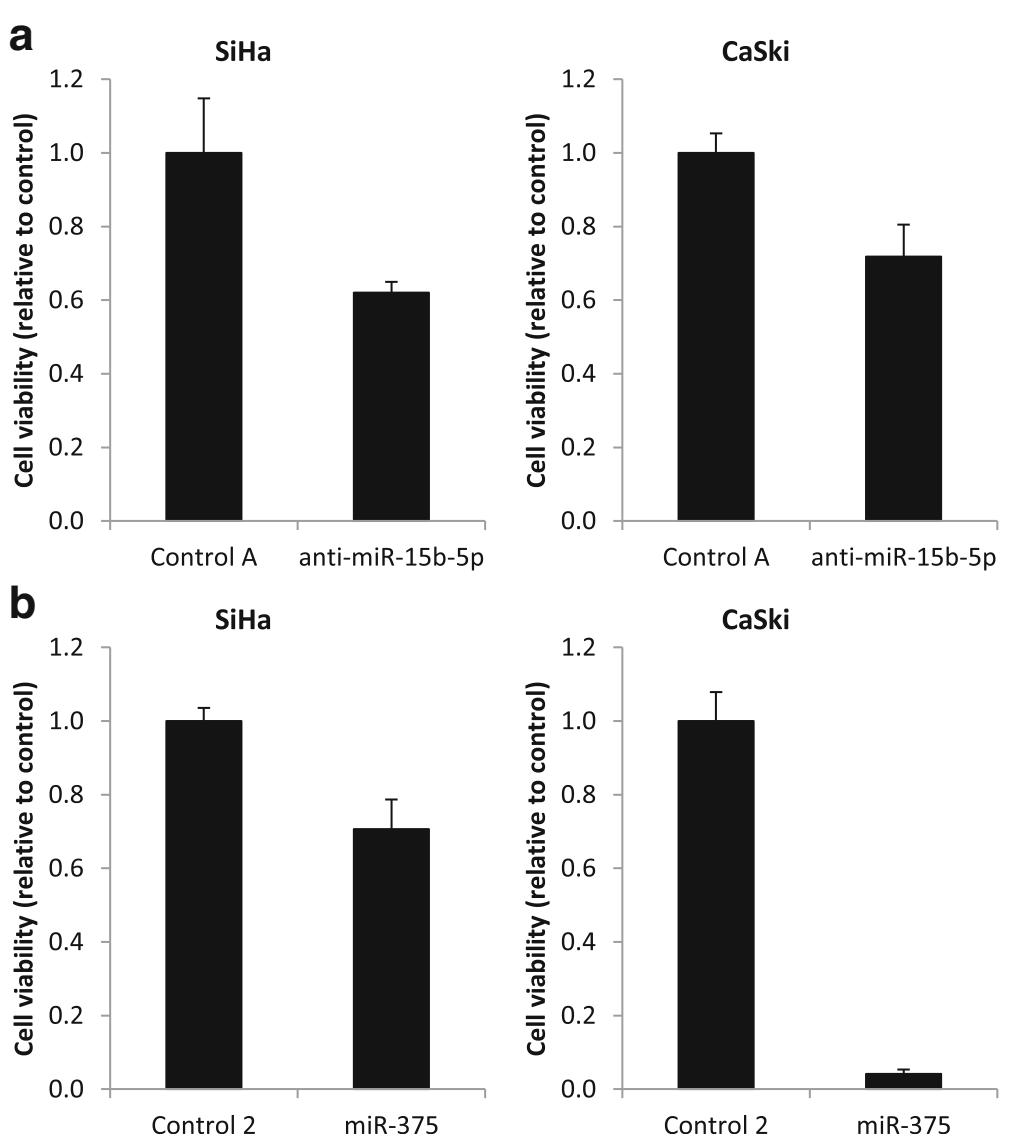

Fig. 4 Functional effect of our selected marker miRNAs in cervical cancer cell lines. Cell viability of SiHa and CaSki cells upon (a) knockdown of miR-15b-5p and (b) ectopic expression of miR-375. Results are representative of two independent experiments

and can be performed in a high-throughput manner. We do acknowledge that further panel optimization is required and expect that analysis of additional miRNAs will result in a miRNA classifier with improved performance. How an optimized miRNA panel performs in comparison to or as adjunct to cytology, HPV16/18 genotyping, DNA methylation markers, and/or other cellular markers such as $\mathrm{p} 16^{\mathrm{INK} 4 \mathrm{~A}} / \mathrm{Ki}-67$ will be subject of future studies.

Overexpression of miR-15b-5p has previously been associated with HPV-induced malignancies including cervical cancer, tonsillar tumors, and anal carcinomas [40, 41]. Moreover, miR-15b-5p was shown to promote cell viability, migration, and invasion in non-small cell lung cancer by targeting metastasis suppressor TIMP2 [42]. In line with this, we observed reduced cervical cancer cell viability upon knockdown of miR-15b-5p suggestive of an oncogenic role for this miRNA. Expression of miR-375 in cervical cancer, on the other hand, is downregulated by increased DNA methylation as well as by a frequently occurring focal loss of chromosome 2q35 [18, 19, 43, 44]. In line with literature, we found that ectopic expression of miR-375 in hrHPV-positive cervical cancer cell lines reduces cell viability [19, 45]. Jung et al. previously showed that miR-375 restored major tumor suppressors p53, p21, and RB levels by deregulation of HPV16 and HPV18 viral transcripts and directly targeting E6AP [45].

One limitation of this study is that we did not include HPV-positive scrapes of women diagnosed with CIN1 and CIN2. Further validation of our 2-miRNA classifier is needed in an independent population-based screening cohort consisting of consecutive hrHPV-positive cervical scrapes including CIN1 and CIN2 lesions. In addition, the samples used in our study had been stored at room temperature for at least 1 year and at $-80{ }^{\circ} \mathrm{C}$ for another 3 to 4 years, and clinical material was limited. We showed that using as little as $20 \mathrm{ng}$ of small RNA still enables the early detection of cervical cancer, but we cannot exclude that higher amounts of RNA, as also used by Tian et al., may give a better discrimination between normal and CIN3. On the other hand, our data also demonstrate that miRNAs are very stable molecules. This is of particular importance in screening settings where cervical scrape material is send to central diagnostic laboratories for molecular testing. While we 
analyzed a selected panel of eight miRNAs which were shown to become genetically or epigenetically deregulated during cervical carcinogenesis in cervical tissue samples, candidate miRNAs should ideally be selected directly from whole miRNome data obtained from cervical scrapes [46]. Future studies will therefore aim to identify an optimal panel of miRNAs for the detection of CIN3 and cancer.

\section{Conclusions}

In conclusion, present data show that analysis of differentially expressed miRNAs may provide an alternative molecular triage strategy in hrHPV-based cervical screening. Our data indicate that miRNAs that are genetically or epigenetically deregulated during and directly involved in disease progression are promising biomarkers for the detection of cervical cancer and a subset of CIN3 lesions. CIN3 detection was further improved by inclusion of HPV16/18 genotyping. Further optimization of the marker panel and validation in an independent cohort of hrHPV-positive cervical scrapes will reveal whether triage of hrHPV-positive women by miRNA expression analysis offers an objective and economical alternative to cytology.

\section{Additional files}

Additional file 1: Figure S1. Correlation between microarray and qRTPCR results for the eight selected miRNAs [17]. Results are shown for cervical tissue specimens of women without disease (normal, $n=9$ ), with precancer (CIN2-3, $n=18$ ), squamous cell carcinoma (SCC, $n=9$ ), and

adenocarcinoma ( $A C, n=9$ ). Linear regression is indicated by the black line and Spearman correlation coefficients (Rho) are shown. QRT-PCR results were normalized to RNU24 and miR-423 and all values were $\log 2$ transformed. (PDF $270 \mathrm{~kb}$ )

Additional file 2: Figure S2. Differential expression of selected miRNAs in cervical tissue specimens. QRT-PCR results were normalized to RNU24 and miR-423, and all values were square root transformed. ${ }^{*} p<0.05,{ }^{* *} p$ $<0.005$. (PDF $179 \mathrm{~kb}$ )

Additional file 3: Table S1. $p$ values of differentially expressed miRNAs in tissue samples. $p$ values were determined by Wilcoxon rank test and corrected applying the Benjamini-Hochberg correction method for multiple testing. QRT-PCR results obtained from normal squamous epithelium ( $(n=8)$, CIN2-3 $(n=18)$, SCC $(n=22)$, and AC $(n=11)$ were included in the analysis. (PDF $262 \mathrm{~kb}$ )

Additional file 4: Table S2. $p$ values of differentially expressed miRNAs in cervical scrapes, $p$ values were determined by Wilcoxon rank test and were corrected by applying the Benjamini-Hochberg correction method for multiple testing. GRT-PCR results obtained from cervical scrapes of women without disease $(n=66)$, CIN2-3 $(n=121)$, SCC $(n=29)$, or AC $(n=9)$ were included in the analysis. (PDF $262 \mathrm{~kb}$ )

\section{Abbreviations}

(hr)HPV: (High-risk) Human papillomavirus; AC: Adenocarcinoma; AUC: Area under the curve; CIN: Cervical intraepithelial neoplasia; E6AP: E6-associated protein; miRNA: MicroRNA; p21: Cyclin dependent kinase inhibitor 1A; p53: Tumor protein p53; RB: Retinoblastoma protein; ROC: Receiver-operated characteristic; SCC: Squamous cell carcinoma

\section{Funding}

This work was supported by the Dutch Cancer Society (KWF VU 2012-5708) and the European Research Council (ERC advanced 2012-AdG, proposal 322986; Mass-Care)

\section{Availability of data and materials}

The datasets produced and analyzed in the current study are available from the corresponding author on reasonable request.

\section{Authors' contributions}

PJFS, CJLMM, RDMS, and SMW were involved in the conception and design of the study. Clinical material collection and data acquisition were carried out by IB, BS, AJ, NVT, DAMH, CJLMM, and SMW. IB, PWN, PJFS, CJLMM, RDMS, and SMW analyzed and interpreted the data. IB, PJFS, RDMS, and SW were major contributors in writing and reviewing the manuscript. All authors read and approved the final manuscript.

\section{Ethics approval and consent to participate}

This study was approved by the Institutional Review Boards of the VU University Medical Center and Antoni van Leeuwenhoek Hospital. All samples were used in an anonymous fashion in accordance with the "Code for Proper Secondary Use of Human Tissues in the Netherlands" as formulated by the Dutch Federation of Medical Scientific Organizations (https://www.federa.org) [47].

\section{Competing interests}

DAMH occasionally serves on the scientific advisory board of Pfizer and Bristol-Meyer Squibb and has been on the speakers' bureau of Qiagen. PJFS, RDMS, CJLMM, and DAMH are minority stakeholders of Self-screen B.V., a spin-off company of VU University Medical Center; and since September 2017, CJLMM is director of Self-screen B.V., which holds patents related to the work. PJFS has received speakers' bureau honoraria from Roche, Qiagen, Gen-Probe, Abbott, and Seegene. He is consultant for Crucell Holland B.V. CJLMM has participated in the sponsored speaker's bureau of Merck and Qiagen and served occasionally on the scientific advisory board of Qiagen and Merck. CJLMM owns a small number of shares of Qiagen, has occasionally been consultant for Qiagen, and until April 2016 was a minority shareholder of Diassay B.V. No potential conflicts of interest were disclosed by the other authors.

\section{Publisher's Note}

Springer Nature remains neutral with regard to jurisdictional claims in published maps and institutional affiliations.

\section{Author details}

${ }^{1}$ Cancer Center Amsterdam, Department of Pathology, VU University Medical Center, Amsterdam, The Netherlands. ${ }^{2}$ Department of Epidemiology and Biostatistics, VU University Medical Center, Amsterdam, The Netherlands. ${ }^{3}$ Center for Gynaecological Oncology, Antoni van Leeuwenhoek Hospital/ Netherlands Cancer Institute, Amsterdam, The Netherlands. ${ }^{4}$ Department of Medical Oncology, Erasmus MC Cancer Institute, Erasmus University Medical Center, Rotterdam, The Netherlands.

Received: 7 November 2017 Accepted: 29 May 2018

Published online: 07 June 2018

\section{References}

1. Peto PJ, Gilham PC, Fletcher O, Matthews FE. The cervical cancer epidemic that screening has prevented in the UK. Lancet. 2004:364:249-56.

2. Walboomers JM, Jacobs MV, Manos MM, Bosch FX, Kummer JA, Shah KV, et al. Human papillomavirus is a necessary cause of invasive cervical cancer worldwide. J Pathol. 1999;189:12-9.

3. Steenbergen RDM, Snijders PJF, Heideman D a M, Meijer CJLM. Clinical implications of (epi)genetic changes in HPV-induced cervical precancerous lesions. Nat Rev Cancer. 2014;14:395-405.

4. Ferlay J, Soerjomataram I, Dikshit R, Eser S, Mathers C, Rebelo M, et al. Cancer incidence and mortality worldwide: sources, methods and major patterns in GLOBOCAN 2012. Int J Cancer. 2015;135:E359-86.

5. Arbyn M, Ronco G, Anttila A, Meijer CJLM, Poljak M, Ogilvie G, et al. Evidence regarding human papillomavirus testing in secondary prevention of cervical cancer. Vaccine. 2012;30(Suppl 5):F88-99. 
6. Ronco G, Dillner J, Elfström KM, Tunesi S, Snijders PJF, Arbyn M, et al. Efficacy of HPV-based screening for prevention of invasive cervical cancer: follow-up of four European randomised controlled trials. Lancet. 2014;383:524-32.

7. Castle PE, Stoler MH, Wright TC, Sharma A, Wright TL, Behrens CM. Performance of carcinogenic human papillomavirus (HPV) testing and HPV16 or HPV18 genotyping for cervical cancer screening of women aged 25 years and older: a subanalysis of the ATHENA study. Lancet Oncol. 2011;12:880-90

8. Cox JT, Castle PE, Behrens CM, Sharma A, Wright TC, Cuzick J, et al. Comparison of cervical cancer screening strategies incorporating different combinations of cytology, HPV testing, and genotyping for HPV 16/18: results from the ATHENA HPV study. Am J Obstet Gynecol. 2013;208:184.e1-184.e11.

9. Nakamura Y, Matsumoto K, Satoh T, Nishide K, Nozue A, Shimabukuro K, et al. HPV genotyping for triage of women with abnormal cervical cancer screening results: a multicenter prospective study. Int J Clin Oncol. 2015;20:974-81.

10. Bartel DP. MicroRNAs: Genomics, biogenesis, mechanism, and Function. Cell. 2004;116:281-97.

11. Li Y, Kowdley KV. MicroRNAs in common human diseases. Genomics Proteomics Bioinformatics. 2012;10:246-53.

12. Mraz M, Malinova K, Mayer J, Pospisilova S. MicroRNA isolation and stability in stored RNA samples. Biochem Biophys Res Commun. 2009;390:1-4.

13. Jung M, Schaefer A, Steiner I, Kempkensteffen C, Stephan C, Erbersdobler A, et al. Robust microRNA stability in degraded RNA preparations from human tissue and cell samples. Clin Chem. 2010;56:998-1006.

14. Chen X, Ba Y, Ma L, Cai X, Yin Y, Wang K, et al. Characterization of microRNAs in serum: a novel class of biomarkers for diagnosis of cancer and other diseases. Cell Res. 2008;18:997-1006.

15. Yu D-C, Li Q-G, Ding X-W, Ding Y-T. Circulating microRNAs: potential biomarkers for cancer. Int J Mol Sci. 2011;12:2055-63.

16. Cheng G. Circulating miRNAs: roles in cancer diagnosis, prognosis and therapy. Adv Drug Deliv Rev. 2015;81:75-93.

17. Wilting SM, Snijders PJF, Verlaat W, Jaspers $A, M$ a $v d$ W, van Wieringen WN, et al. Altered microRNA expression associated with chromosomal changes contributes to cervical carcinogenesis. Oncogene. 2013;32:106-16.

18. Wilting SM, Verlaat W, Jaspers A, Makazaji NA, Agami R, Meijer CJ, et al. Methylation-mediated transcriptional repression of microRNAs during cervical carcinogenesis. Epigenetics. 2013:8:220-8.

19. Bierkens M, Krijgsman O, Wilting SM, Bosch L, Jaspers A, Meijer GA, et al. Focal aberrations indicate EYA2 and hsa-miR-375 as oncogene and tumor suppressor in cervical carcinogenesis. Genes Chromosomes Cancer. 2013;52:56-68.

20. Hesselink AT, Berkhof J, van der Salm ML, van Splunter AP, Geelen TH, van Kemenade FJ, et al. Clinical validation of the HPV-risk assay, a novel realtime PCR assay for detection of high-risk human papillomavirus DNA by targeting the E7 region. J Clin Microbiol. 2014;52:890-6.

21. Geraets DT, Cuschieri K, de Koning MNC, van Doorn LJ, Snijders PJF, Meijer CJLM, et al. Clinical evaluation of a GP5+/6+-based luminex assay having full high-risk human papillomavirus genotyping capability and an internal control. J Clin Microbiol. 2014:52:3996-4002.

22. Polman NJ, Oštrbenk A, Xu L, Snijders PJF, Meijer CJLM, Poljak M, et al. Evaluation of the clinical performance of the HPV-risk assay using the VALGENT-3 panel. J Clin Microbiol. 2017;55:3544-51.

23. Colgan TJ, Lickrish GM. The topography and invasive potential of cervical adenocarcinoma in situ, with and without associated squamous dysplasia. Gynecol Oncol. 1990;36:246-9.

24. Bekkers RLM, Bulten J, Wiersma-van Tilburg A, Mravunac M, Schijf CPT, Massuger LFAG, et al. Coexisting high-grade glandular and squamous cervical lesions and human papillomavirus infections. Br J Cancer. 2003;89:886-90.

25. Babion I, Snoek BC, van de Wiel MA, Wilting SM, Steenbergen RDM. A strategy to find suitable reference genes for miRNA quantitative PCR analysis and its application to cervical specimens. J Mol Diagnostics. 2017; 19:625-37.

26. Livak KJ, Schmittgen TD. Analysis of relative gene expression data using real-time quantitative PCR and the 2(-Delta Delta C(T)) method. Methods. 2001;25:402-8

27. DeLong ER, DeLong DM, Clarke-Pearson DL. Comparing the areas under two or more correlated receiver operating characteristic curves: a nonparametric approach. Biometrics. 1988;44:837-45.

28. Snellenberg S, Cillessen SAGM, Van Criekinge W, Bosch L, Meijer CJLM, Snijders PJF, et al. Methylation-mediated repression of PRDM14 contributes to apoptosis evasion in HPV-positive cancers. Carcinogenesis. 2014;35:2611-8.

29. Peccoud J, Jacob C. Theoretical uncertainty of measurements using quantitative polymerase chain reaction. Biophys J. 1996;71:101-8.
30. Bengtsson M, Hemberg M, Rorsman P, Ståhlberg A. Quantification of mRNA in single cells and modelling of RT-qPCR induced noise. BMC Mol Biol. 2008;9:63.

31. Korenková V, Scott J, Novosadová V, Jindřichová M, Langerová L, Švec D, et al. Pre-amplification in the context of high-throughput QPCR gene expression experiment. BMC Mol Biol. 2015;16:5.

32. Rijkaart DC, Berkhof J, Van Kemenade FJ, Coupe VMH, Hesselink AT, Rozendaal $L$, et al. Evaluation of 14 triage strategies for HPV DNA-positive women in population-based cervical screening. Int J Cancer. 2011;130:602-310.

33. Sharma G, Dua P, Agarwal SM. A comprehensive review of dysregulated miRNAs involved in cervical Cancer. Curr Genomics. 2014;15:310-23.

34. Li Y, Wang F, Xu J, Ye F, Shen Y, Zhou J, et al. Progressive miRNA expression profiles in cervical carcinogenesis and identification of HPV-related target genes for miR-29. J Pathol. 2011;224:484-95.

35. Zeng K, Zheng W, Mo X, Liu F, Li M, Liu Z, et al. Dysregulated microRNAs involved in the progression of cervical neoplasm. Arch Gynecol Obstet. 2015;292:905-13.

36. Tian $Q, L i Y$, Wang F, Li Y, X J, Shen Y, et al. MicroRNA detection in cervical exfoliated cells as a triage for human papillomavirus-positive women. J Natl Cancer Inst. 2014;106 https://doi.org/10.1093/jnci/dju241.

37. Hesselink AT, Sahli R, Berkhof J, Snijders PJF, van der Salm ML, Agard D, et al. Clinical validation of Anyplex ${ }^{\text {TM }}$ II HPV HR detection according to the guidelines for HPV test requirements for cervical cancer screening. J Clin Virol. 2016;76:36-9.

38. De Strooper LMA, Meijer CJLM, Berkhof J, Hesselink AT, Snijders PJF, Steenbergen RDM, et al. Methylation analysis of the FAM19A4 gene in cervical scrapes is highly efficient in detecting cervical carcinomas and advanced CIN2/3 lesions. Cancer Prev Res. 2014;7:1251-7.

39. Dijkstra MG, van Niekerk D, Rijkaart DC, van Kemenade FJ, Heideman DAM, Snijders PJF, et al. Primary hrHPV DNA testing in cervical Cancer screening: how to manage screen-positive women? A POBASCAM trial substudy. Cancer Epidemiol Biomark Prev. 2014;23:55-63.

40. Vojtechova Z, Sabol I, Salakova M, Smahelova J, Zavadil J, Turek L, et al. Comparison of the miRNA profiles in HPV-positive and HPV-negative tonsillar tumors and a model system of human keratinocyte clones. BMC Cancer. 2016;16:382.

41. Myklebust M, Bruland O, Fluge $\varnothing$, Skarstein A. MicroRNA-15b is induced with E2F-controlled genes in HPV-related cancer. Br J Cancer. 2011;105: $1719-25$.

42. Wang H, Zhan Y, Jin J, Zhang C, Li W. MicroRNA-15b promotes proliferation and invasion of non-small cell lung carcinoma cells by directly targeting TIMP2. Oncol Rep. 2017;37:3305-12.

43. Liu S, Song L, Yao H, Zhang L, Xu D, Gao F, et al. MiR-375 is epigenetically downregulated by HPV-16 E6 mediated DNMT1 upregulation and modulates EMT of cervical cancer cells by suppressing IncRNA MALAT1. PLoS One. 2016;11:e0163460

44. Morel A, Baguet A, Perrard J, Demeret C, Jacquin E, Guenat D, et al. 5azadC treatment upregulates miR-375 level and represses HPV16 E6 expression. Oncotarget. 2017:8:46163-76.

45. Jung H, Phillips BL, Chan EK. miR-375 activates p21 and suppresses telomerase activity by coordinately regulating HPV E6/E7, E6AP, CIP2A, and 14-3-3ろ. Mol Cancer. 2014;13:80

46. Novianti PW, Snoek BC, Wilting SM, van de Wiel MA. Better diagnostic signatures from RNAseq data through use of auxiliary co-data. Bioinformatics. 2017;33:1572-4.

47. Federa. Human Tissue and Medical Research: Code of conduct for responsible use (2011). Rotterdam: Federa; 2011. http://www.bbmri.nl/wpcontent/uploads/2015/10/Federa_code_of_conduct_english.pdf. Accessed 25 Oct 2017. 